\title{
Suppression of Heat Shock Protein 27 Using OGX-427 Induces Endoplasmic Reticulum Stress and Potentiates Heat Shock Protein 90 Inhibitors to Delay Castrate-resistant Prostate Cancer
}

\author{
François Lamoureux ${ }^{1}$, Christian Thomas ${ }^{1}$, Min-Jean Yin ${ }^{2}$, Ladan Fazli ${ }^{1}$, Amina Zoubeidi ${ }^{1}$, \\ and Martin E. Gleave ${ }^{1, *}$ \\ ${ }^{1}$ The Vancouver Prostate Centre, University of British Columbia, Vancouver, BC, Canada \\ ${ }^{2}$ Oncology Research, Pfizer Worldwide Research \& Development, San Diego, CA, USA
}

\begin{abstract}
Background-Although prostate cancer responds initially to androgen ablation therapies, progression to castration-resistant prostate cancer (CRPC) frequently occurs. Heat shock protein (Hsp) 90 inhibition is a rational therapeutic strategy for CRPC that targets key proteins such as androgen receptor (AR) and protein kinase B (Akt); however, most Hsp90 inhibitors trigger elevation of stress proteins like Hsp27 that confer tumor cell survival and treatment resistance.

Objective-We hypothesized that cotargeting the cytoprotective chaperone Hsp27 and Hsp90 would amplify endoplasmic reticulum (ER) stress and treatment-induced cell death in cancer.

Design, setting, and participants-Inducible and constitutive Hsp27 and other HSPs were measured by real-time reverse transcription-polymerase chain reaction and immunoblot assays. The combinations of OGX-427 with Hsp90 inhibitors were evaluated in vitro for LNCaP cell growth and apoptosis and in vivo in CRPC LNCaP xenograft models.
\end{abstract}

\footnotetext{
(C) 2013 European Association of Urology. Published by Elsevier B.V. All rights reserved. Fax: +1 604875 5654. m.gleave@ubc.ca (M. Gleave). the resulting proof before it is published in its final citable form. Please note that during the production process errors may be discovered which could affect the content, and all legal disclaimers that apply to the journal pertain. and the accuracy of the data analysis.

Study concept and design: Gleave, Zoubeidi, Yin, Lamoureux.

Acquisition of data: Lamoureux, Thomas, Fazli.

Analysis and interpretation of data: Gleave, Zoubeidi, Yin, Lamoureux.

Drafting of the manuscript: Gleave, Zoubeidi, Lamoureux.

Critical revision of the manuscript for important intellectual content: Gleave, Zoubeidi, Lamoureux.

Statistical analysis: Lamoureux, Thomas, Fazli.

Obtaining funding: Gleave, Zoubeidi, Yin.

Administrative, technical, or material support: Gleave, Zoubeidi, Lamoureux.

Supervision: Gleave, Zoubeidi.

Other (specify): None.
}

"Corresponding author. The Vancouver Prostate Centre, 2660 Oak Street, Vancouver, BC, V6H 3Z6, Canada. Tel.: +1 604 875 5686;

Publisher's Disclaimer: This is a PDF file of an unedited manuscript that has been accepted for publication. As a service to our customers we are providing this early version of the manuscript. The manuscript will undergo copyediting, typesetting, and review of

Author contributions: Martin E. Gleave had full access to all the data in the study and takes responsibility for the integrity of the data 
Outcome measurements and statistical analysis-Tumor volumes were compared using the Kruskal-Wallis test. Overall survival was analyzed using Kaplan-Meier curves, and statistical significance was assessed with the log-rank test.

Results and limitations-Hsp90 inhibitors induced expression of HSPs in tumor cells and tissues in a dose- and time-dependent manner; in particular, Hsp27 mRNA and protein levels increased threefold. In vitro, OGX-427 synergistically enhanced Hsp90 inhibitor-induced suppression of cell growth and induced apoptosis by $60 \%$ as measured by increased sub-G1 fraction and poly(ADP-ribose) polymerase cleavage. These biologic events were accompanied by decreased expression of HSPs, Akt, AR, and prostate-specific antigen, and induction of ER stress markers (cleaved activating transcription factor 6, glucose-regulated protein 78, and DNAdamage-inducible transcript 3). In vivo, OGX-427 potentiated the anticancer effects of Hsp90 inhibitor PF-04929113 (orally, $25 \mathrm{mg} / \mathrm{kg}$ ) to inhibit tumor growth and prolong survival in CRPC LNCaP xenografts.

Conclusions-HSP90 inhibitor-mediated induction of Hsp27 expression can be attenuated by OGX-427, resulting in increased ER stress and apoptosis, and synergistic inhibition of CRPC tumor growth.

Patient summary-This study supports the development of targeted strategies using OGX-427 in combination with Hsp90 inhibitors to improve patient outcome in CRPC.

\section{Keywords}

OGX-427; Hsp27; Hsp90 inhibition; Androgen receptor; Castration-resistant prostate cancer

\section{Introduction}

Although early detection and treatment of localized prostate cancer (PCa) has improved survival rates, many men still die of metastatic disease. Although $80 \%$ of patients initially respond to androgen-deprivation therapy, most progress to metastatic castrate-resistant prostate cancer (CRPC) [1,2]. To significantly improve survival in men with PCa, new therapeutic strategies targeting the molecular basis of CRPC and treatment resistance are required.

Molecular chaperones including heat shock proteins (HSPs) help tumor cells cope with stress-induced misfolded proteins and play prominent roles in cellular signaling and transcriptional regulatory networks. In particular, Hsp90 and Hsp27 are highly expressed in various cancers, including CRPC, and induce tumor cell survival or treatment resistance [3]. Hsp90 is an ATPase-dependent chaperone required for protein folding, maturation, and conformational stabilization of many client proteins [4]. Hsp90 interacts with several proteins involved in CRPC including growth factor receptors, cell cycle regulators, and signaling kinases, including protein kinase B (Akt) or androgen receptor (AR) [5]. Tumor cells express higher Hsp90 levels and activity than benign cells [6,7], and Hsp90 inhibition has emerged as a target in CRPC and other cancers. Many Hsp90 inhibitors were developed that target the ATPase pocket, including natural compounds such as geldanamycin and its analog 17-allylamino-17-demethoxy-geldanamycin (17-AAG), or synthetic compounds including PF-04928473. These agents inhibited Hsp90 function and induced apoptosis in 
preclinical studies of cancers of the colon, breast, and prostate, among others [7,8]. While promising, treatment resistance emerges early due to compensatory mechanisms involving activation of heat shock factor (HSF) 1, which induces increased expression of HSPs, including Hsp70 and clusterin [9]. Interestingly, the upregulation of these chaperones plays a role in cellular recovery from stress by restoring protein homeostasis and promoting thermotolerance and cell survival [10]. Among them, Hsp27 is a stress-activated chaperone that interacts with many key apoptosisassociated proteins to regulate a cell's apoptotic rheostat through both intrinsic and extrinsic pathways. We previously reported that knocking down Hsp27 using a specific inhibitor, OGX-427, induces apoptosis and potentiates the effect of anticancer drugs both in vitro and in vivo in CRPC and bladder cancer [11]. OGX-427 is currently in a multicenter phase 2 clinical trial in CRPC and metastatic bladder cancer (NCT01454089 and NCT01120470) [12,13].

Molecular chaperones play key roles in endoplasmic reticulum (ER) stress responses, thereby regulating protein homeostasis. Disruption of proteostasis induces ER stress, which, in turn, leads to the unfolded protein response (UPR), a prosurvival process induced to restore normal ER function. The UPR is distinguished by the action of three signaling proteins localized on the ER membrane: pancreatic ER kinase (PKR)-like ER kinase (PERK), inositol requiring enzyme (IRE) 1, and activating transcription factor (ATF) 6 that are kept inactive through the association of their luminal domain with the ER chaperone binding immunoglobulin protein/glucose-regulated protein (BiP/GRP) 78 [14]. Increasing levels of misfolded proteins in the ER lumen release the three ER stress sensors from BiP/ GRP78, allowing the activation of their signaling functions and the transcription of UPR target genes such as activating transcription factor (ATF) 4, X-box binding protein (XBP) 1 , and DNA-damage-inducible transcript 3 (CHOP). However, excessive ER stress leads to mitochondrial apoptosis to eliminate damaged cells [15], which is mainly controlled by the pro-apoptotic transcription factor CHOP [16]. Therefore, cotargeting molecular chaperones regulating ER homeostasis may enhance cancer control by overwhelming a cancer cell's ability to regulate misfolded protein burden. In this regard, Hsp90 modulates the UPR by interacting and stabilizing two of three ER stress sensors, IRE1 and PERK [17], so that Hsp90 inhibition induces ER-stress-mediated apoptosis [18,19]. We recently reported that Hsp27 plays an important role in ER homeostasis and that knocking down Hsp27 using OGX-427 induces ER stress [11]. We set out to test the hypothesis that Hsp90 inhibition induction of $\mathrm{Hsp} 27$ functions to inhibit treatment-induced apoptosis and enhance emergence of treatment resistance. It follows that cotargeting Hsp27 (using OGX-427) will potentiate effects of Hsp90 inhibitors (17-AAG and PF-04928473) by amplifying ER stress and leading to apoptosis in CRPC.

\section{Materials and methods}

\subsection{Cell culture experiments}

The human PCa cell lines PC-3 and LNCaP were maintained in Dulbecco modified Eagle medium and Roswell Park Memorial Institute medium 1640, respectively (Life Technologies Corp, Carlsbad, CA, USA) supplemented with 5\% fetal bovine serum and 2 $\mathrm{mmol} / \mathrm{L}_{\mathrm{L}}$ glutamine in a humidified $5 \%$ carbon dioxide/air atmosphere at $37^{\circ} \mathrm{C}$. Hsp90 
inhibitor PF-04928473 [4-(6,6-dimethyl-4-oxo-3-trifluoromethyl-4,5,6,7-tetrahydroindazol-1-yl)-2-(4-hydroxy-cyclohexylamino)-benzamide] and its prodrug PF-04929113 (Pfizer Inc, New York, NY, USA) were used for in vitro and in vivo studies, respectively. These compounds are synthetic inhibitors that bind the N-terminal adenosine triphosphate binding site of Hsp90; PF-04929113 is orally bioavailable. Stocks and dilutions of PF-04928473 and 17-AAG (US National Institutes of Health, Bethesda, MD, USA) were made in dimethyl sulfoxide. Cells were quantified using crystal violet staining. The sequence of OGX-427 (OncoGenex Pharmaceuticals, Bothell, WA, USA) corresponds to the human Hsp27 translation initiation site (5'-GGGACGCGGCGCTCGGTCAT-3'). Scrambled antisense oligonucleotide (ScrB ASO; ISIS Pharmaceuticals, Carlsbad, CA, USA) was used as a control.

\subsection{Protein detection}

Western blots were probed with antibodies anti-Hsp70, anti-AR, anti-PSA, anti-GRP78, anti-ATF4, and anti-CHOP (Santa Cruz Biotechnology, Dallas, TX, USA); anti-phosphoeIF2a (Life Technologies Corp, Carlsbad, CA, USA); anti-ATF6 (ImgenexCorp, San Diego, CA, USA); anti-HSP27 (Enzo Life Sciences Inc, Farmingdale, NY, USA); anti-PARP and anti-Akt (Cell Signaling Technology Inc, Beverly, MA, USA); and anti-vinculin (SigmaAldrich Corp, St. Louis, MO, USA). For immunohistochemistry, paraffin-embedded, formalin-fixed tissue sections were antigen retrieved, incubated with the primary antibody followed by secondary antibodies (Life Technologies Corp, Carlsbad, CA, USA).

\subsection{RNA quantification}

For real-time quantitative polymerase chain reaction, total RNA was extracted and cDNA quantified by the ABI PRISM 7900HT Sequence Detection System (Life Technologies Corp, Carlsbad, CA, USA). Relative expression was calculated using the delta-Ct method.

\subsection{Animal treatment}

For xenograft experiments, $2 \times 10^{6} \mathrm{LNCaP}$ cells (suspended in $0.1 \mathrm{ml}$ Matrigel; Becton, Dickinson and Co, Franklin Lakes, NJ, USA) were rapidly injected subcutaneously into the flank region of athymic male mice (Harlan Sprague-Dawley, Inc, Indianapolis, IN, USA). The mice were castrated once tumors reached between $300 \mathrm{~mm}^{3}$ and $500 \mathrm{~mm}^{3}$ or the prostate-specific antigen (PSA) level increased $>50 \mathrm{ng} / \mathrm{ml}$. Once tumors progressed to castrate resistance, mice were randomly assigned to vehicle PF-04929113, PF-04929113 plus ScrB ASO, or PF-04929113 plus OGX-427. Tumor volume was measured twice weekly. All animal procedures were performed according to the guidelines of the Canadian Council on Animal Care and appropriate institutional certification.

\subsection{Statistical analysis}

All in vitro data were assessed using the Student $t$ test and Mann-Whitney test. Tumor volumes of mice were compared using the Kruskal-Wallis test. Overall survival was analyzed using Kaplan-Meier curves, and statistical significance was assessed with the logrank test. Levels of statistical significance were set at $p<0.05$. Additional methods are presented in the Supplement. 


\section{Results}

\subsection{Heat shock protein 90 inhibitors induce heat shock protein expression in prostate cancer}

Both 17-AAG and PF-04928473 increased Hsp27 and Hsp70 protein levels up to threefold in a dose- and time-dependent manner in LNCaP and PC-3 cells (Fig. 1a-1c) and were accompanied by a decline of total Akt expression. mRNA levels of Hsp27, Hsp70, and Hsp90 also increased after Hsp90 inhibitor treatment (Fig. 1d).

Next, the effects of PF-04928473 on Hsp27 expression in CRPC LNCaP xenografts were assessed using immunohistochemistry (Fig. 2). Once tumors became palpable, mice were treated with Hsp90 inhibitors. Hsp27 expression increased 2.5-fold after treatment with PF-04929113 compared with vehicle (Fig. 2). Similarly, Hsp70, a pharmacodynamic measure of Hsp90 inhibition [8,20], increased 2.3-fold after treatment with PF-04929113 (Fig. 2).

\subsection{Heat shock protein 27 protects tumor cells from heat shock protein 90 inhibition- induced endoplasmic reticulum stress}

Since Hsp90 inhibition using PF-04928473 or 17-AAG induces Hsp27 expression (Fig. 1 and 2), Hsp27 overexpression protects tumor cells from Hsp90 inhibitor treatment (Fig. 3a), and key proteins initiating ER stress, such as PERK or IRE1, are client proteins of Hsp90 [17]. We consequently analyzed if PF-04928473 induces ER stress and UPR in PCa cell lines (Fig.3b and 3c). PF-04928473 treatment increased expression of the ER resident chaperone Grp78, a marker of ER stress induction (Fig. 3c). Hsp90 inhibition induced upregulation of ATF4, involved in the PERK signaling of the UPR, and upregulation of XBP1 splicing, suggesting IRE1 and/or ATF6 signaling activation (Fig. 3c). CHOP expression, a major component of the ER stress-mediated apoptosis, was also increased after Hsp90 inhibitor treatment. These results indicate that Hsp90 inhibition induces ER stress in PCa cells by activating several signaling pathways of the UPR associated with the upregulation of Hsp27 expression (Fig. 3c).

We previously reported that Hsp27 plays an important role in ER stress regulation [11]. The proteasome inhibitor MG132 is known to induce ER stress by inhibiting the UPR [21]. MG132 treatment induced ER stress and UPR by increasing Grp78, p-eIF2a, ATF4, cleaved ATF6, and CHOP expression (Fig. 3d, Supplemental Fig. 1a). This upregulation of ER stress and UPR signaling was also associated with increased Hsp27 expression. Hsp27 induction was also observed after Hsp90 or proteasome inhibitor treatments (Fig.3c and 3d, Supplemental Fig. 1a), which both lead to ER stress and UPR induction, identifying Hsp27 as a key component in the ER stress and UPR.

We next evaluated the effect of OGX-427-induced Hsp27 knockdown on ER stress and UPR induction. Interestingly, OGX-427 induced both ER stress and UPR activation by increasing Grp78, p-eIF2a, ATF4, cleaved ATF6, and CHOP expression, as well as XBP1 splicing (Fig. 3e, Supplemental Fig. 1b), suggesting a protective role of Hsp27 in protein homeostasis. 


\subsection{Cotargeting heat shock protein 90 and heat shock protein 27 amplifies endoplasmic reticulum stress and treatment-induced apoptosis}

Since Hsp90 inhibitors induce Hsp27, and Hsp27 functions as a mediator of ER stress regulation and treatment resistance [22], we next evaluated if Hsp27 knockdown potentiated the effect of Hsp90 inhibition. Combined Hsp27 (using OGX-427) and Hsp90 (with PF-04928473 or 17-AAG) inhibition induced Grp78 and higher levels of CHOP and XBP1 splicing compared with control or each drug alone (Fig. 4a and 4b, Supplemental Fig. 2a). To assess synergy, combination index (CI) values were calculated according to the Chou and Talalay median effect principle. Dose-response curves show that OGX-427 plus PF-04929113 or OGX-427 plus 17-AAG treatments more potently inhibited tumor cell growth than monotherapy (Fig. 4c). The CI curves and plots (lower than one) indicate that OGX-427 synergistically enhanced the effect of both Hsp90 inhibitors on tumor cell growth (Fig. 4c) and induction of apoptosis. Flow cytometric analysis showed that apoptotic rates (subG1 fraction) increased significantly when OGX-427 was combined with PF-04929113 (38\%) compared with control ScrB (4.5\%), OGX-427 (8.7\%), or control ScrB plus PF-04928473 (25.8\%) (Fig. 4d). Enhanced apoptosis was confirmed by the caspase-3 activity assay and cleaved-PARP expression (Fig. 4e, 4f, Supplemental Fig. 2b). In addition, OGX-427 blocked PF-094928473-induced Hsp27 expression and decreased Akt, AR, and PSA expression when combined with Hsp90 inhibition in LNCaP cells (Fig. 4f). Collectively, these data confirm that cotargeting Hsp27 and Hsp90 amplified ER stress, overwhelming adaptive responses to increase stress-induced apoptosis.

\subsection{OGX-427 potentiates PF-04929113 activity in LNCaP castrate-resistant prostate cancer xenografts}

The effects of combined treatment with OGX-427 and PF-04929113 were evaluated in LNCaP CRPC xenografts. All animals treated with OGX-427 plus PF-04929113 $(n=8)$ had significant delays in tumor growth compared with other groups starting at day 7 (respectively, $209.9 \mathrm{~mm}^{3}$ and $906.2 \mathrm{~mm}^{3}$ for control, and $473.2 \mathrm{~mm}^{3}$ for PF-04929113 and $590.2 \mathrm{~mm}^{3}$ for PF-04929113 plus control ScrB) and after $45 \mathrm{~d}$ (respectively, $527.2 \mathrm{~mm}^{3}$ and $2883.7 \mathrm{~mm}^{3}$ for control, and $2130.4 \mathrm{~mm}^{3}$ for PF-04929113 and $1892.9 \mathrm{~mm}^{3}$ for PF-04929113 plus ScrB) (Fig. 5a). Moreover, combination OGX-427 plus PF-04929113 significantly decreased tumor progression (138.2\%) (Fig. 5b) compared with other groups (control: 827.3\%; PF-04929113: 655.6\%; and PF-04929113: 438.6\%).

Serum PSA levels were also significantly lower (approximately 2.5 -fold) in mice receiving OGX-427 plus PF-04929113 compared with either drug alone (Fig. 5c). The combination OGX-427 plus PF-04929113 group had mean PSA levels of $186.4 \mathrm{ng} / \mathrm{ml}$ after $49 \mathrm{~d}$ compared with $445.7 \mathrm{ng} / \mathrm{ml}$ in the vehicle group, $510 \mathrm{ng} / \mathrm{ml}$ in the PF-04929113 group, or $431.6 \mathrm{ng} / \mathrm{ml}$ in the scrB plus PF-4929113 group. The combination OGX-427 plus PF-04929113 group had a significantly increased PSA doubling time (14.49 wk) and decreased PSA velocity (23.02 ng/ml per week) compared with other groups (PSA doubling time: approximately $1.7-2.4 \mathrm{wk}$; velocity: approximately $70.32-94.85 \mathrm{ng} / \mathrm{ml}$ per week) (Fig. 5d). 
Overall survival was significantly prolonged in mice treated with combined OGX-427 plus PF-04929113 (all mice were alive after 56 d) compared with control (none of eight alive after 52 d), PF-04929113 (none of eight alive after 52 d), or ScrB plus PF-04929113 (two of eight alive after $52 \mathrm{~d} ; p<0.001$ ) (Fig. 5e).

Immunohistochemical analysis indicated decreased Hsp27, Grp78, Ki67, and AR expression after treatment with combined OGX-427 plus PF-04929113 compared with other groups (Fig.6a and 6b), corroborating the in vitro results. Additionally, tumors treated with combination OGX-427 plus PF-04929113 had significantly higher apoptosis rates compared with other groups, as shown by increased terminal deoxynucleotidyl transferase dUTP nick end labeling (TUNEL) staining (Fig.6a and 6b), suggesting that decreases in progression of OGX-427 plus PF-04929113 tumors resulted from both reduced proliferation rates and increased apoptosis rates.

\section{Discussion}

Although several Hsp90 inhibitors, including PF-04928473, have potent antitumor activity in preclinical models [23], human trials have shown little clinical activity. One explanation is that Hsp90 inhibitors induce a stress response involving HSF-1 and subsequent increased levels of Hsp90 itself, as well as Hsp70, Hsp27, and clusterin (CLU) [9,24]. This induction of HSPs may confer acquired resistance to Hsp90 inhibitors. Development of treatment resistance is a common feature of solid tumor malignancies and the underlying basis for most cancer deaths. Treatment resistance evolves, in part, from selective pressures of treatment that collectively increase the apoptotic rheostat of cancer cells. Survival proteins upregulated after apoptotic triggers include antiapoptotic members of the Bcl-2 protein family, CLU, HSPs, and survivin [25]. In fact, inhibition of Hsp90, Hsp70, Hsp27, or CLU induces cell death and sensitization to chemotherapy [23,26].

Chaperones act as buffers to stabilize the phenotype of various cells and organisms at times of environmental stress, and they enhance the Darwinian fitness of cells during progression and treatment resistance [27]. Indeed, the heat shock response is associated with oncogenic transformation, proliferation, survival, and thermotolerance [28]. Thus targeting molecular chaperones like Hsp90 and Hsp27 with multifunctional roles in ER stress, cellular signaling, and transcriptional regulatory networks associated with cancer progression and treatment resistance is an attractive and rational therapeutic strategy.

In the present study, we focused on Hsp27, which is induced by hormone therapy or chemotherapy and inhibits treatment-induced apoptosis through multiple mechanisms [3,29,30]. Hsp27 is highly expressed in CRPC [31] and involved in various pathophysiologic processes such as survival and apoptosis [30]. Hsp27 overexpression has been associated with chemoresistance, and with radiation and hormone resistance [32], making Hsp27 an attractive target for antitumor therapeutics. Consistent with these findings, inhibition of Hsp27 using OGX-427 synergistically enhances conventional as well as targeted therapies in prostate and other cancers [33]. Furthermore, OGX-427 is currently in multicenter phase 2 clinical trials in CRPC (NCT01681433 and NCT01120470) [34,35] combined with chemotherapy. 
We confirm that Hsp90 inhibition induces ER stress and HSP expression including Hsp27. Treatment with Hsp90 inhibitors robustly increases the ER resident chaperone Grp78 as well as ATF4, sXBP1, and CHOP, indicating that Hsp90 inhibitors activate different arms of the ER/UPR pathway [19]. Upregulation of CHOP, a transcription factor and major mediator of the ER stress/UPR pathway, suggests that Hsp90 inhibition induces apoptosis at least in part by activating the ER stress/UPR pathway. However, Hsp90 inhibition also increases Hsp27 expression, which functions to protect tumor cells from Hsp90 inhibitor-induced apoptosis. We confirmed that increased Hsp27 levels reduce PF-04928473-induced ER stress and accumulation of misfolded protein levels through mechanisms involving increased UPR activity. Overexpression of Hsp27 attenuates ER stress and protects tumor cells from apoptosis by maintaining protein homeostasis and reducing unfolded protein burden [11]. We show that Hsp27 knockdown affects the three ER stress sensors: (1) the PERK pathway, by increasing p-eIF2 $a$ and ATF4; (2) the IRE1 pathway, by increasing sXBP1; and (3) theATF6 pathway, by enhancing cleaved ATF6, consistent with our previous study [11]. Interestingly, combining OGX-427 and Hsp90 inhibitors led to prolonged ER stress by blocking treatment resistance induced by Hsp27, overwhelming unfolded protein response, as shown by significant increased $\mathrm{CHOP}$ expression, and leading to apoptosis.

Consequently, cotargeting Hsp90 and Hsp27, both involved in the ER stress/UPR pathway, is a rational therapeutic strategy for $\mathrm{PCa}$.

Combining OGX-427 with an HSP90 inhibitor reduced cell proliferation while increasing apoptotic rates. Consistent with these in vitro results, synergistic effects of combined treatment were also observed in vivo in an LNCaP xenograft model. Systemic administration of OGX-427 plus PF-04929113 inhibited tumor growth and prolonged survival compared with control plus PF-04929113 in LNCaP CRPC. Detection of increased TUNEL staining with combined therapy suggests that delayed tumor progression resulted, in part, from enhanced apoptosis. Combination of OGX-427 with PF-0492113 also reduced PSA levels in the LNCaP CRPC model. Serum PSA level is an established AR-regulated biomarker and a valuable tool in assessing anticancer activity. Hsp90 inhibition is known to destabilize and degrade the AR with decreased PSA expression [8]. In vivo, serum PSA levels as well as PSA doubling time and velocity were significantly reduced with combination OGX-427 therapy compared with PF-04929113 monotherapy, consistent with decreased AR immunostaining. Interestingly, at the low doses of Hsp90 inhibitor used in this study, no effect on serum PSA level was apparent. However, combination OGX-427 plus PF-04929113 disturbed AR stability and lowered PSA levels, suggesting that Hsp90 inhibitor-induced Hsp27 may stabilize AR and its transcriptional activity as reported in the literature [36]. Combined inhibition of Hsp27 and Hsp90 leads to AR degradation and decreased expression of its target genes, like PSA.

A potential advantage of combining two drugs is to reduce toxicity by using lower doses of each single drug. Hsp90 inhibitors are known to induce severe hepatotoxicity, especially $17-$ AAG used as monotherapy at $60 \mathrm{mg} / \mathrm{kg}$ [37]. In our study, we used $25 \mathrm{mg} / \mathrm{kg}$ PF-04929113 as the therapeutic dose, without any side effects observed. At this dose, PF-04929113 monotherapy showed marginal and nonsignificant decreases in tumor volume without affecting serum PSA level. Decrease in tumor volume was significantly apparent only when PF-04929113 was combined with OGX-427. 


\section{Conclusions}

This paper helps define how stress induced by Hsp90 inhibitors regulates Hsp27 and, in turn, how Hsp27 regulates ER stress and UPR components, cell survival, and treatment resistance. These observations are clinically relevant because Hsp27 inhibitors are in phase 2 clinical trials and provide a framework for building new drug combinations based on mechanism-based interventions to overcome drug resistance. This study provides the first support for the development of targeted strategies using OGX-427 in combination with Hsp90 inhibitors to improve patient outcome in CRPC.

\section{Supplementary Material}

Refer to Web version on PubMed Central for supplementary material.

\section{Acknowledgments}

Acknowledgment statement: We thank Gerrit Los for his support and discussion, and Virginia Yago and Estelle Li for technical assistance.

Financial disclosures: Martin E. Gleave certifies that all conflicts of interest, including specific financial interests and relationships and affiliations relevant to the subject matter or materials discussed in the manuscript (eg, employment/ affiliation, grants or funding, consultancies, honoraria, stock ownership or options, expert testimony, royalties, or patents filed, received, or pending), are the following: The University of British Columbia has submitted patent applications, listing M. Gleave as inventor, on the antisense sequence described in this paper. This intellectual property has been licensed to OncoGenex Technologies, a Vancouver-based biotechnology company in which M. Gleave has founding shares. M.-J. Yin is an employee of Pfizer.

Funding/Support and role of the sponsor: This study was supported by Pfizer Worldwide Research \& Development (USA), the Pacific Northwest Prostate SPORE, L'Association pour la Recherche sur le Cancer (France), and the Canadian Institutes of Health Research.

\section{References}

1. Gleave ME, Goldenberg SL, Chin JL, et al. Randomized comparative study of 3 versus 8-month neoadjuvant hormonal therapy before radical prostatectomy: biochemical and pathological effects. J Urol. 2001; 166:500-506. discussion 506-7. [PubMed: 11458055]

2. Gleave M, Goldenberg SL, Bruchovsky N, Rennie P. Intermittent androgen suppression for prostate cancer: rationale and clinical experience. Prostate Cancer Prostatic Dis. 1998; 1:289-296. [PubMed: 12496869]

3. Rocchi P, Beraldi E, Ettinger S, et al. Increased Hsp27 after androgen ablation facilitates androgenindependent progression in prostate cancer via signal transducers and activators of transcription 3mediated suppression of apoptosis. Cancer Res. 2005; 65:11083-11093. [PubMed: 16322258]

4. Young JC, Hartl FU. Polypeptide release by Hsp90 involves ATP hydrolysis and is enhanced by the co-chaperone p23. EMBO J. 2000; 19:5930-5940. [PubMed: 11060043]

5. Takayama S, Reed JC, Homma S. Heat-shock proteins as regulators of apoptosis. Oncogene. 2003; 22:9041-9047. [PubMed: 14663482]

6. Chiosis G, Huezo H, Rosen N, Mimnaugh E, Whitesell L, Neckers L. 17AAG: low target binding affinity and potent cell activity—finding an explanation. Mol Cancer Ther. 2003; 2:123-129. [PubMed: 12589029]

7. Kamal A, Thao L, Sensintaffar J, et al. A high-affinity conformation of Hsp90 confers tumour selectivity on Hsp90 inhibitors. Nature. 2003; 425:407-410. [PubMed: 14508491]

8. Solit DB, Zheng FF, Drobnjak M, et al. 17-Allylamino-17-demethoxygeldanamycin induces the degradation of androgen receptor and HER-2/neu and inhibits the growth of prostate cancer xenografts. Clin Cancer Res. 2002; 8:986-993. [PubMed: 12006510] 
9. Lamoureux F, Thomas C, Yin MJ, et al. Clusterin inhibition using OGX-011 synergistically enhances Hsp90 inhibitor activity by suppressing the heat shock response in castrate-resistant prostate cancer. Cancer Res. 2011; 71:5838-5849. [PubMed: 21737488]

10. Zoubeidi A, Zardan A, Wiedmann RM, et al. Hsp27 promotes insulin-like growth factor-I survival signaling in prostate cancer via p90Rsk-dependent phosphorylation and inactivation of BAD. Cancer Res. 2010; 70:2307-2317. [PubMed: 20197463]

11. Kumano M, Furukawa J, Shiota M, et al. Cotargeting stress-activated Hsp27 and autophagy as a combinatorial strategy to amplify endoplasmic reticular stress in prostate cancer. Molecular Cancer Ther. 2012; 11:1661-1671.

12. [Updated July 13, 2013] A randomized, double-blind phase 2 study comparing gemcitabine and cisplatin in combination with OGX-427 or placebo in patients with advanced transitional cell carcinoma. ClinicalTrials.gov Web site. http://clinicaltrials.gov/show/NCT01454089.

13. [Updated February 21, 2013] A randomized phase II study of OGX-427 (a second-generation antisense oligonucleotide to heat shock protein-27) in patients with castration resistant prostate cancer who have not previously received chemotherapy for metastatic disease. ClinicalTrials.gov Web site. http://clinicaltrials.gov/ct2/show/NCT01120470?term=NCT01120470\&rank=1.

14. Ron D, Harding HP. Protein-folding homeostasis in the endoplasmic reticulum and nutritional regulation. Cold Spring Harb Perspect Biol. 2012; 4

15. Hetz C. The unfolded protein response: controlling cell fate decisions under ER stress and beyond. Nat Rev Mol Cell Biol. 2012; 13:89-102. [PubMed: 22251901]

16. Tabas I, Ron D. Integrating the mechanisms of apoptosis induced by endoplasmic reticulum stress. Nat Cell Biol. 2011; 13:184-190. [PubMed: 21364565]

17. Marcu MG, Doyle M, Bertolotti A, Ron D, Hendershot L, Neckers L. Heat shock protein 90 modulates the unfolded protein response by stabilizing IRE1alpha. Mol Cell Biol. 2002; 22:85068513. [PubMed: 12446770]

18. Davenport EL, Moore HE, Dunlop AS, et al. Heat shock protein inhibition is associated with activation of the unfolded protein response pathway in myeloma plasma cells. Blood. 2007; 110:2641-2649. [PubMed: 17525289]

19. Gallerne C, Prola A, Lemaire C. Hsp90 inhibition by PU-H71 induces apoptosis through endoplasmic reticulum stress and mitochondrial pathway in cancer cells and overcomes the resistance conferred by Bcl-2. Biochim Biophys Acta. 2013; 1833:1356-1366. [PubMed: 23485394]

20. Eccles SA, Massey A, Raynaud FI, et al. NVP-AUY922: a novel heat shock protein 90 inhibitor active against xenograft tumor growth, angiogenesis, and metastasis. Cancer Res. 2008; 68:28502860. [PubMed: 18413753]

21. Park HS, Jun do Y, Han CR, Woo HJ, Kim YH. Proteasome inhibitor MG132-induced apoptosis via ER stress-mediated apoptotic pathway and its potentiation by protein tyrosine kinase p56lck in human Jurkat T cells. Biochem Pharmacol. 2011; 82:1110-1125. [PubMed: 21819973]

22. Gleave M, Chi KN. Knock-down of the cytoprotective gene, clusterin, to enhance hormone and chemosensitivity in prostate and other cancers. Ann N Y Acad Sci. 2005; 1058:1-15. [PubMed: 16394121]

23. Lamoureux F, Thomas C, Yin M, et al. A novel HSP90 inhibitor delays castrate resistant prostate cancer without altering serum PSA levels and inhibits osteoclastogenesis. Clin Cancer Res. 2011; 17:2301-2313. [PubMed: 21349995]

24. Cervantes-Gomez F, Nimmanapalli R, Gandhi V. Transcription inhibition of heat shock proteins: a strategy for combination of 17-allylamino-17-demethoxygeldanamycin and actinomycin $\mathrm{d}$. Cancer Res. 2009; 69:3947-3954. [PubMed: 19383903]

25. Gleave ME, Monia BP. Antisense therapy for cancer. Nat Rev Cancer. 2005; 5:468-479. [PubMed: 15905854]

26. Guo F, Rocha K, Bali P, et al. Abrogation of heat shock protein 70 induction as a strategy to increase antileukemia activity of heat shock protein 90 inhibitor 17-allylamino-demethoxy geldanamycin. Cancer Res. 2005; 65:10536-10544. [PubMed: 16288046]

27. Whitesell L, Lindquist SL. HSP90 and the chaperoning of cancer. Nature Rev Cancer. 2005; 5:761-772. [PubMed: 16175177] 
28. Dai C, Whitesell L, Rogers AB, Lindquist S. Heat shock factor 1 is a powerful multifaceted modifier of carcinogenesis. Cell. 2007; 130:1005-1018. [PubMed: 17889646]

29. Garrido C, Fromentin A, Bonnotte B, et al. Heat shock protein 27 enhances the tumorigenicity of immunogenic rat colon carcinoma cell clones. Cancer Res. 1998; 58:5495-5499. [PubMed: 9850085]

30. Garrido C, Bruey JM, Fromentin A, Hammann A, Arrigo AP, Solary E. HSP27 inhibits cytochrome c-dependent activation of procaspase-9. FASEB J. 1999; 13:2061-2070. [PubMed: 10544189]

31. Bubendorf L, Kolmer M, Kononen J, et al. Hormone therapy failure in human prostate cancer: analysis by complementary DNA and tissue microarrays. J Natl Cancer Inst. 1999; 91:1758-1764. [PubMed: 10528027]

32. July LV, Beraldi E, So A, et al. Nucleotide-based therapies targeting clusterin chemosensitize human lung adenocarcinoma cells both in vitro and in vivo. Mol Cancer Ther. 2004; 3:223-232. [PubMed: 15026542]

33. Rocchi P, Jugpal P, So A, et al. Small interference RNA targeting heat-shock protein 27 inhibits the growth of prostatic cell lines and induces apoptosis via caspase-3 activation in vitro. BJU Int. 2006; 98:1082-1089. [PubMed: 16879439]

34. [Updated September 23, 2013] The Pacific Clinical Trial: a randomized phase II study evaluating OGX-427 in patients with metastatic castrate-resistant prostate cancer (MCRPC) who have prostate-specific antigen (PSA) progression while receiving abiraterone: Hoosier Oncology Group GU12-159. ClinicalTrials.gov Web site. http://clinicaltrials.gov/show/NCT01681433.

35. [Updated February 21, 2013] A randomized phase II study of OGX-427 (a second-generation antisense oligonucleotide to heat shock protein-27) in patients with castration resistant prostate cancer who have not previously received chemotherapy for metastatic disease. ClinicalTrials.gov Web site. http://clinicaltrials.gov/ct2/show/NCT01120470?term=NCT01120470\&rank=1.

36. Zoubeidi A, Zardan A, Beraldi E, et al. Cooperative interactions between androgen receptor (AR) and heat-shock protein 27 facilitate AR transcriptional activity. Cancer Res. 2007; 67:1045510465. [PubMed: 17974989]

37. Glaze ER, Lambert AL, Smith AC, et al. Preclinical toxicity of a geldanamycin analog, 17(dimethylaminoethylamino)-17-demethoxygeldanamycin (17-DMAG), in rats and dogs: potential clinical relevance. Cancer Chemother Pharmacol. 2005; 56:637-647. [PubMed: 15986212] 


\section{Take-home message}

This study found that targeting heat shock protein (Hsp) 27 using OGX-427 synergistically enhanced Hsp90 inhibitor activity, delayed tumor progression, and prolonged survival in castrate-resistant prostate cancer. These effects were accompanied with decreased levels of circulating prostate-specific antigen and increased cell apoptosis. 
A

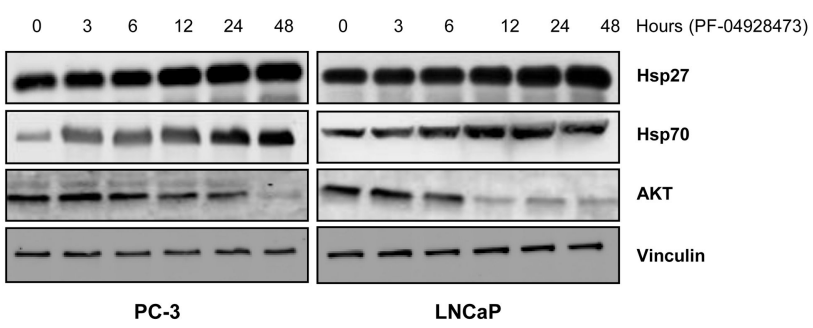

C

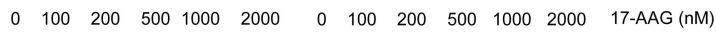

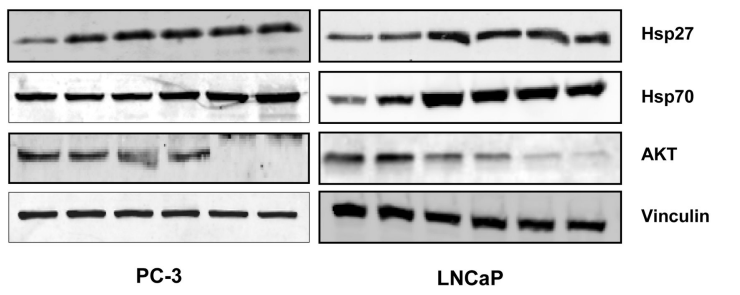

B

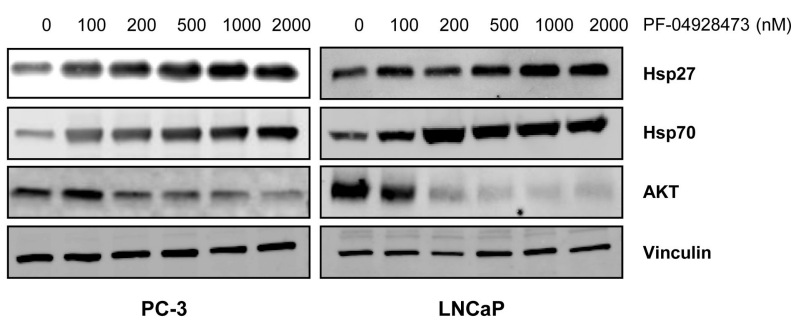

D

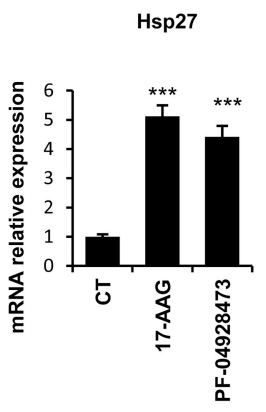

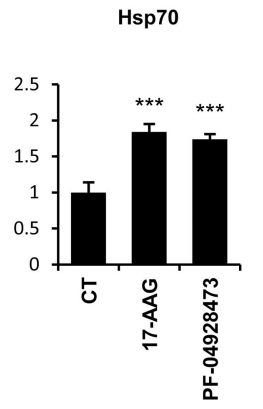

Fig. 1.

PF-04929113 and 17-AAG induce heat shock protein (HSP) expression in prostate cancer cells in vitro. PC-3 and LNCaP cells were treated with (a) $1 \mu \mathrm{M}$ PF-04928473 or (c) $1 \mu \mathrm{M}$ 17-AAG for the indicated time points. (b) In parallel, PC-3 and LNCaP cells were treated for $48 \mathrm{~h}$ with PF-04928473 for the indicated doses. Protein extracts were analyzed for Hsp27, Hsp70, and protein kinase B (Akt) by Western blot; vinculin was used as a loading control. (d) LNCaP tumor cells were treated for $24 \mathrm{~h}$ with $1 \mu \mathrm{M}$ PF-04928473 or $1 \mu \mathrm{M}$ 17-AAG. mRNA extracts were analyzed by real-time polymerase chain reaction for Hsp27, Hsp90, and Hsp70. $* * * p<0.001$. CT $=$ control. 


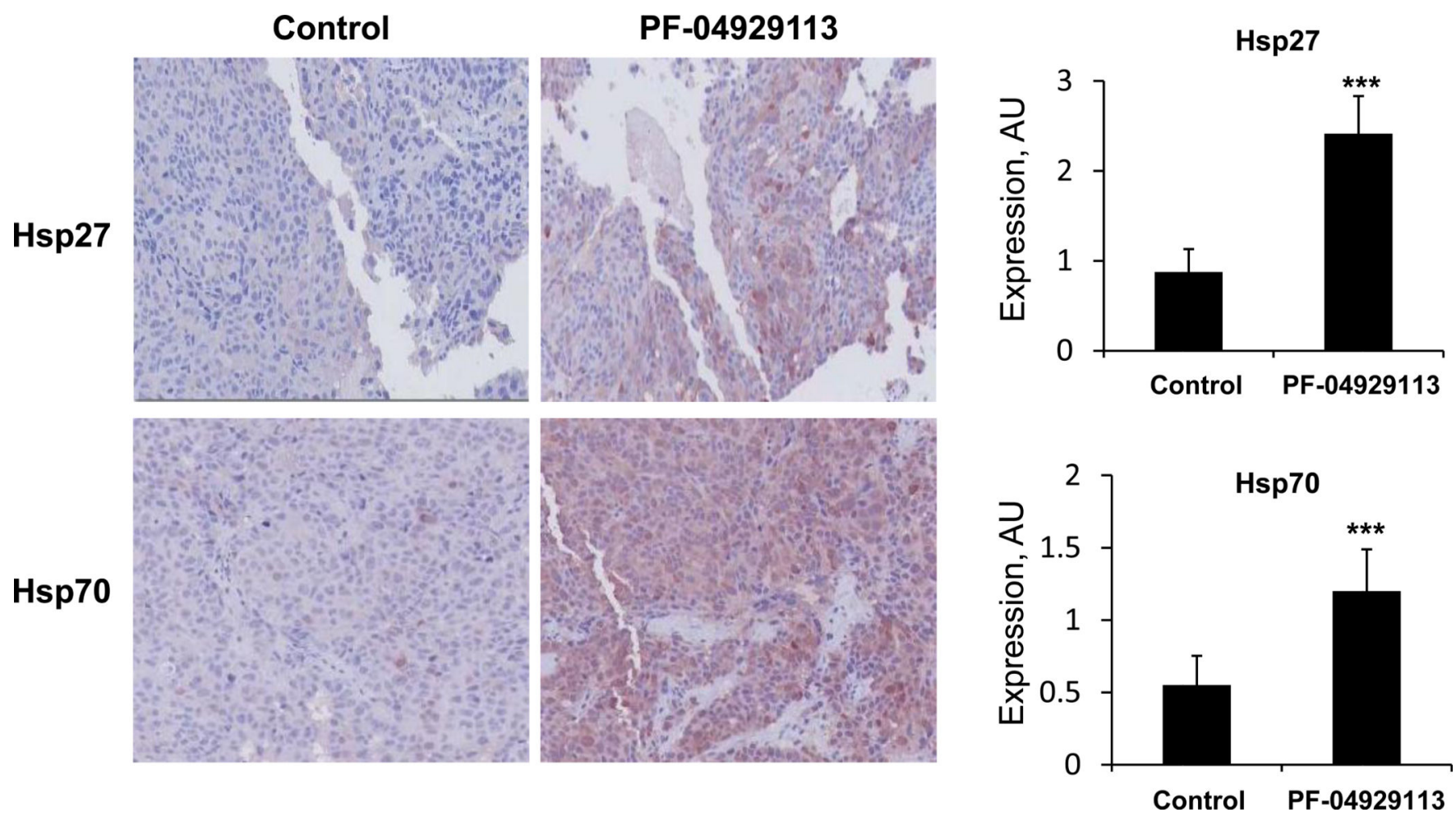

Fig. 2.

PF-04928473 induces heat shock protein (HSP) expression in a prostate cancer xenograft model. Mice were treated for 6 wk with $50 \mathrm{mg} / \mathrm{kg}$ PF-04929113 or vehicle (control). Tumors were collected, and Hsp27 and Hsp70 were evaluated by immunohistochemical analysis. Specimens were scored and estimated in arbitrary units (AUs). *** $p<0.001$. 
A

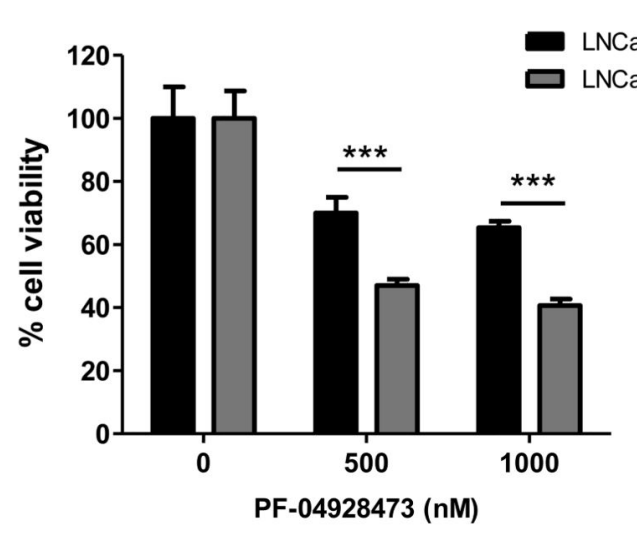

C

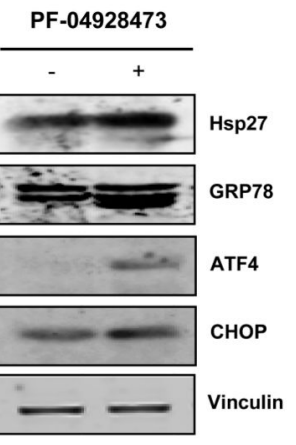

D

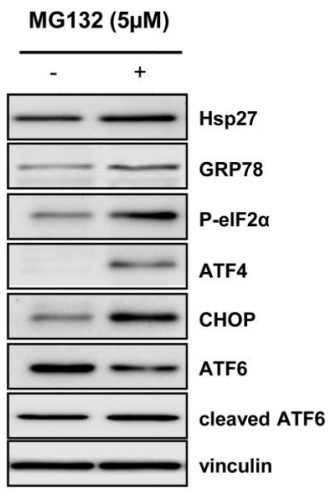

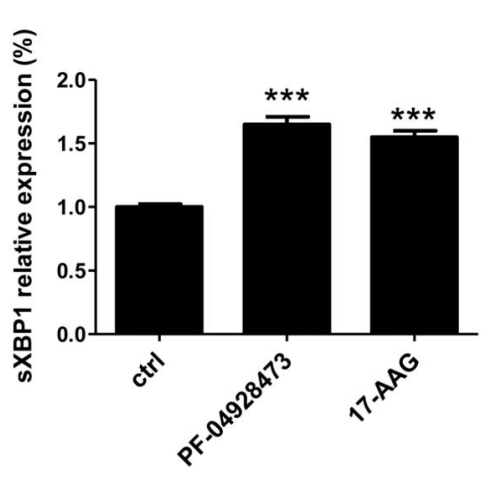

E
B

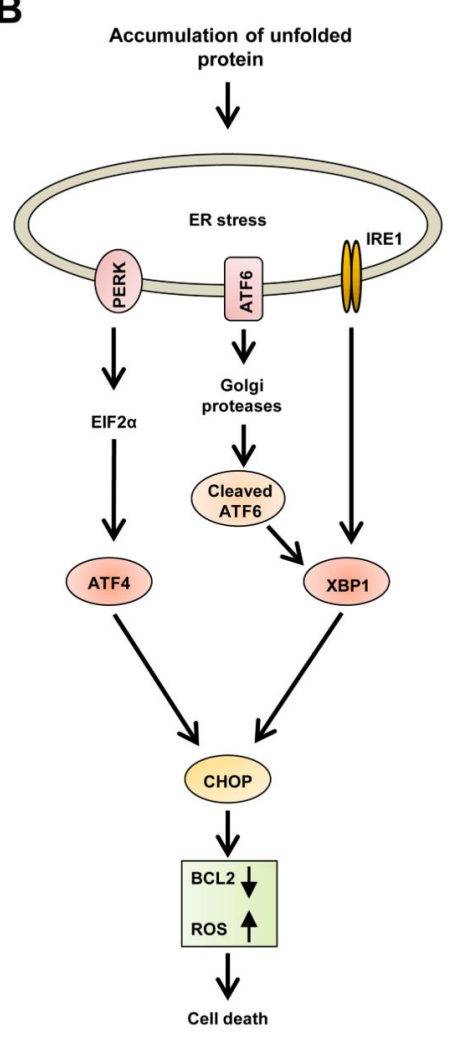

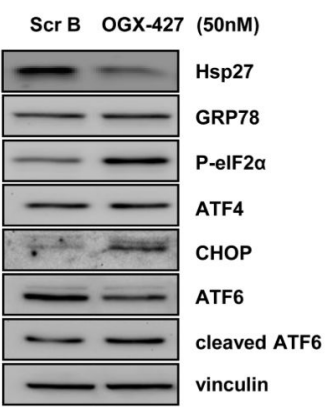

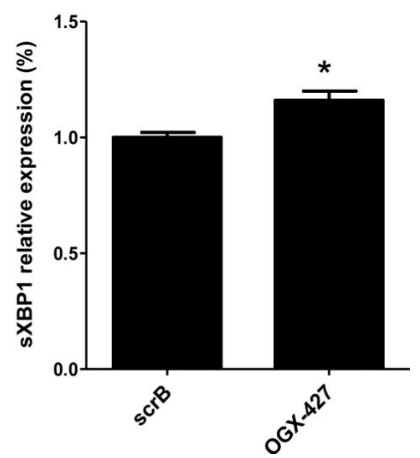

Fig. 3.

Heat shock protein (Hsp) 27 protects tumor cells from Hsp90 inhibition-induced endoplasmic reticulum (ER) stress. (a) Hsp27 overexpressing stable LNCaP cells (LNCaPHsp27) and control vector-transfected cells (LNCaP-Empty) were treated with indicated concentrations of PF-04928473 for $48 \mathrm{~h}$. Cell growth was determined by crystal violet staining. (b) Schematic picture of the ER stress/unfolded protein response pathway. (c) LNCaP cells were treated with $1 \mu \mathrm{M}$ PF-04928473 or 17-AAG for $24 \mathrm{~h}$ (real-time polymerase chain reaction [PCR]) or $48 \mathrm{~h}$ (Western blot). Cells were harvested; Western 
blotting analyses were performed on the cell lysates for Hsp27, glucose-regulated protein (Grp) 78, activating transcription factor (ATF) 4, DNA-damage-inducible transcript 3 (CHOP), and vinculin; or quantitative real-time PCR analysis was performed on RNA for splicing X-box binding protein (sXBP) 1. (d) LNCaP cells were treated with $5 \mu \mathrm{M}$ carbobenzoxy-Leu-Leu-leucinal (MG132) for $48 \mathrm{~h}$. Western blotting analyses were performed for Hsp27, Grp78, P-eiF2a, ATF4, CHOP, ATF6, cleaved ATF6, and vinculin expression. (e) LNCaP cells were treated twice with $50 \mathrm{nM}$ OGX-427 or control ScrB ASO. Cells were harvested, and Western blotting analyses were performed for indicated antibodies or quantitative real-time PCR analysis for sXBP1. Means of at least three independent experiments were done in triplicate. $* * * p<0.001 ; * p<0.05$. IRE1 $=$ inositol requiring enzyme 1; BCL2 = B-cell CLL/lymphoma 2; ROS = c-ros oncogene 1, receptor tyrosine kinase. 
A

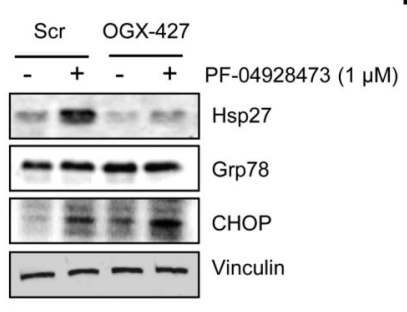

C

$\underline{P F-04928473}$
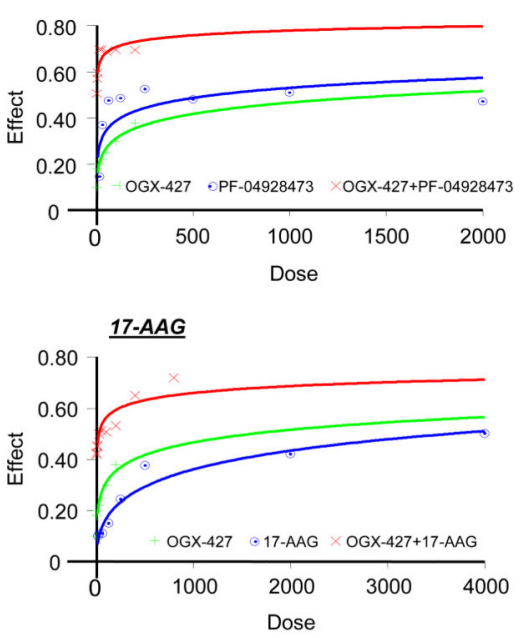

D

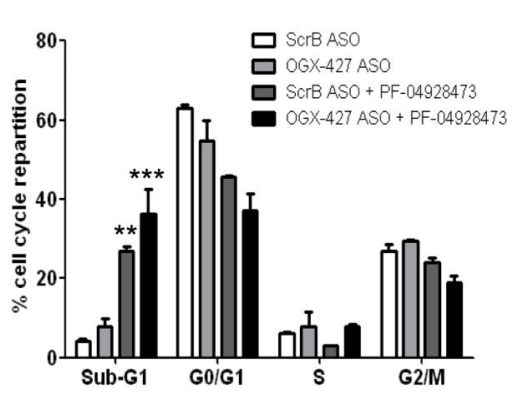

B
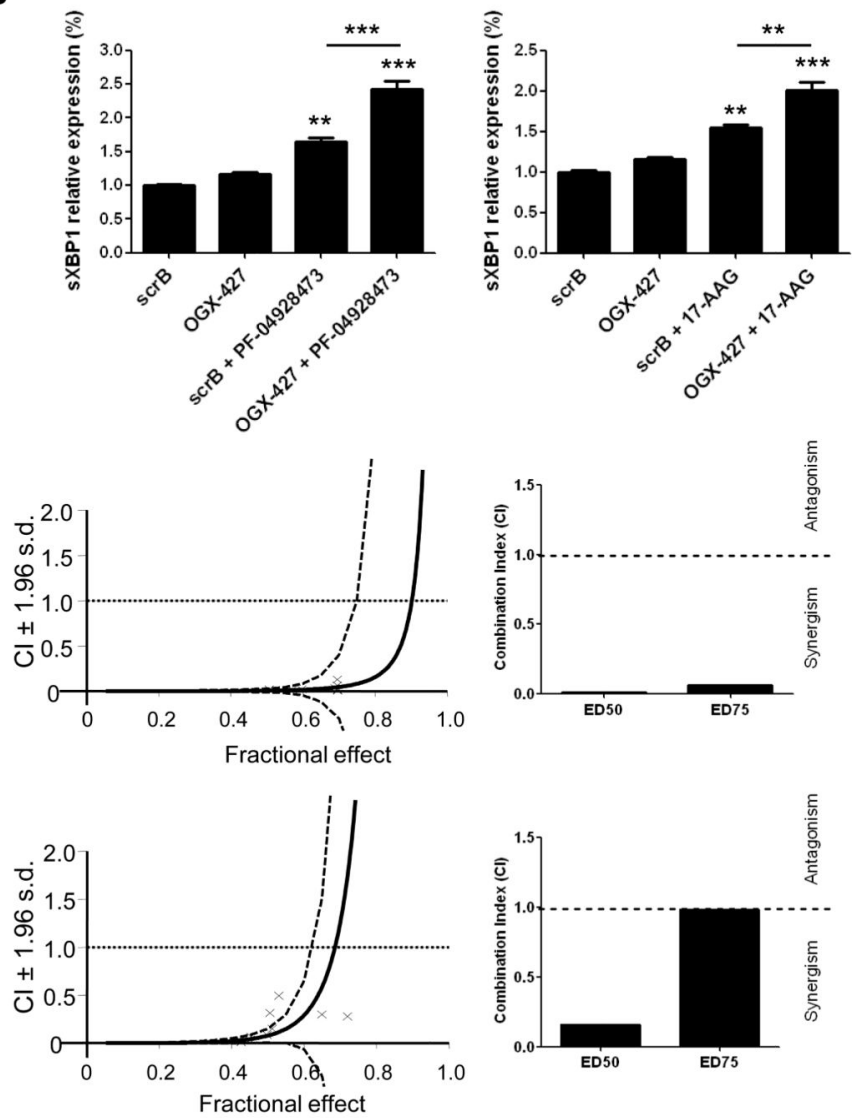

$\mathbf{E}$

$\mathbf{F}$

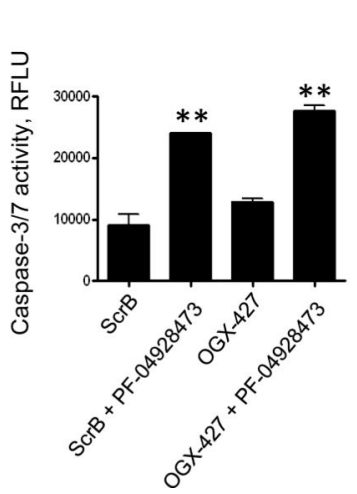

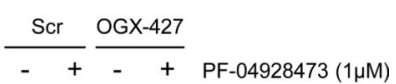

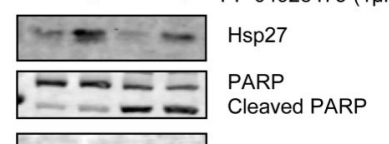

$-=0$ Akt

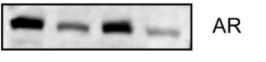

$\infty \quad 2 \quad$ PSA

$\longrightarrow$ Vinculin

Fig. 4.

Cotargeting heat shock protein (Hsp) 90 and Hsp27 amplifies endoplasmic reticulum (ER) stress response and treatment-induced apoptosis. $\mathrm{LNCaP}$ cells were treated twice with 50 nM OGX-427 or control ScrB ASO, followed by $1 \mu$ M PF-04928473 for 48 h. (a) Cells were harvested and Western blotting analyses were performed for Hsp27, glucose-regulated protein (Grp) 78, DNA-damage-inducible transcript 3 (CHOP), and vinculin. (b) Cells were harvested and quantitative real-time polymerase chain reaction analysis was performed on RNA for splicing X-box binding protein (sXBP) 1. (c) Dose-dependent effects and 
combination index (CI) values were assessed in LNCaP cells treated for $48 \mathrm{~h}$ with OGX-427 alone, PF-04928473 or 17-AAG alone, or with combined treatment at the indicated concentration with a constant ratio design between both drugs. The $\mathrm{CI}$ values for effective dose (ED) $)_{50}$ and $\mathrm{ED}_{75}$ were, respectively, 0.05 and 0.1 for PF-04928473, and 0.18 and 0.95 for 17-AAG, indicating a synergistic effect $(<1)$ of Hsp27 inhibition combined with Hsp90 inhibitors. (d-f) LNCaP cells were treated twice with $50 \mathrm{nM}$ OGX-427 or control ScrB ASO, followed by $1 \mu \mathrm{M}$ PF-04928473 for $48 \mathrm{~h}$. (d) The proportion of cells in subG ${ }_{1}, \mathrm{G}_{0}-\mathrm{G}_{1}$, $\mathrm{S}, \mathrm{G}_{2}-\mathrm{M}$ was determined by propidium iodide staining. Cells were harvested, caspase-3 activity was determined on the cell lysates, and the results expressed in arbitrary units (AUs) and corrected for (e) protein content, or (f) Western blotting analyses were performed for Hsp27, poly ADP ribose polymerase (PARP), protein kinase B (AKT), androgen receptor (AR), prostate-specific antigen (PSA), and vinculin expression. All experiments were repeated at least three times. ${ }^{* * *} p<0.001 ; * * p<0.01$. s.d. $=$ standard deviation; RFLU $=$ relative fluorescence unit. 


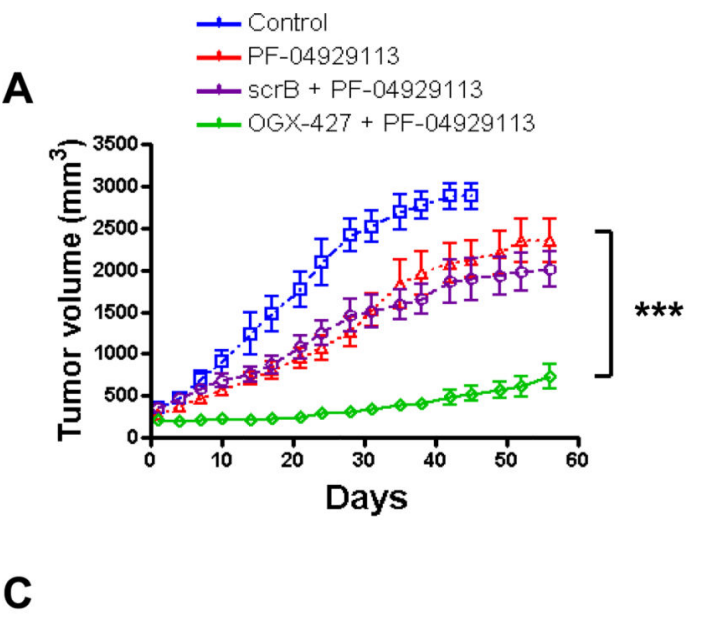

B
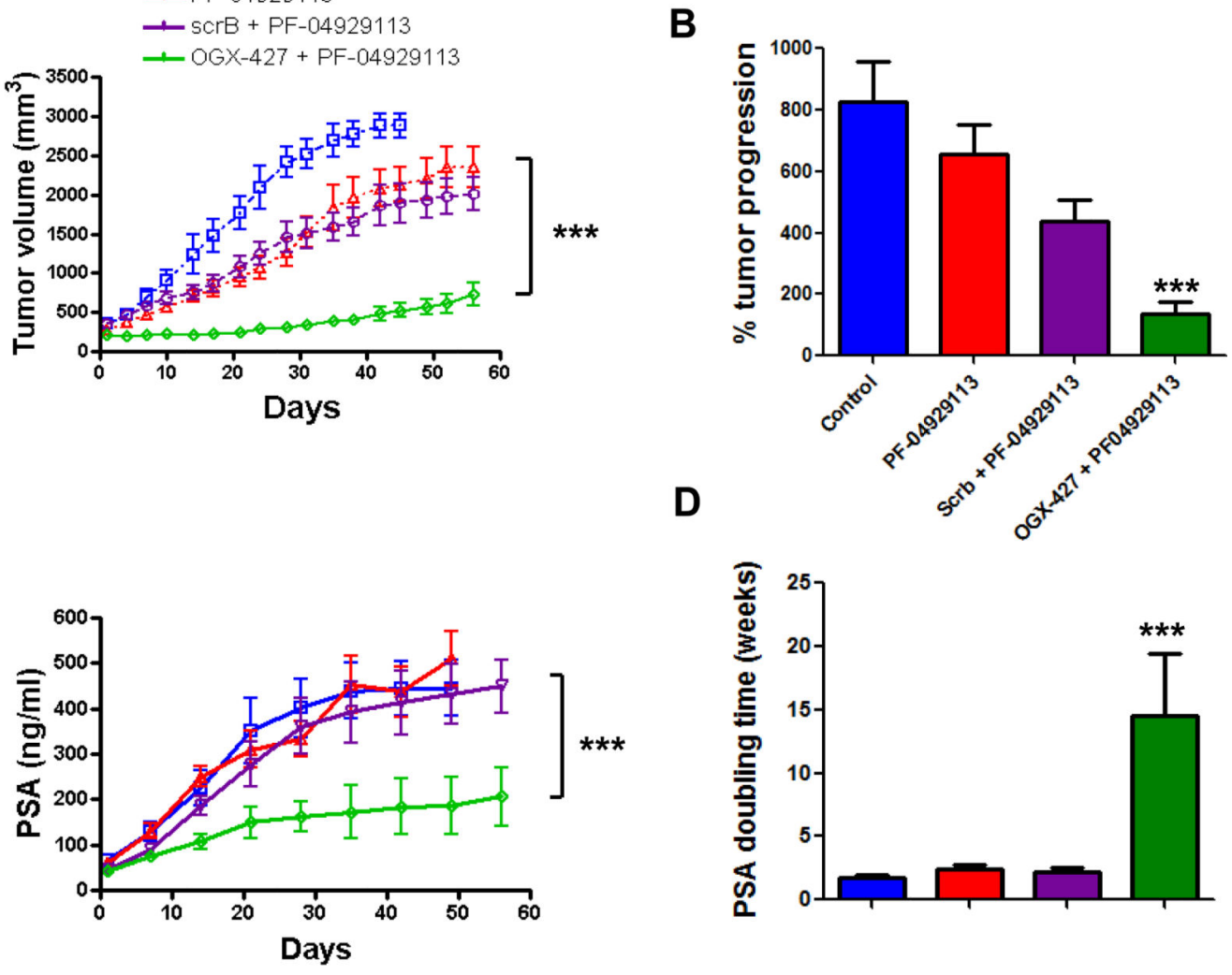

E
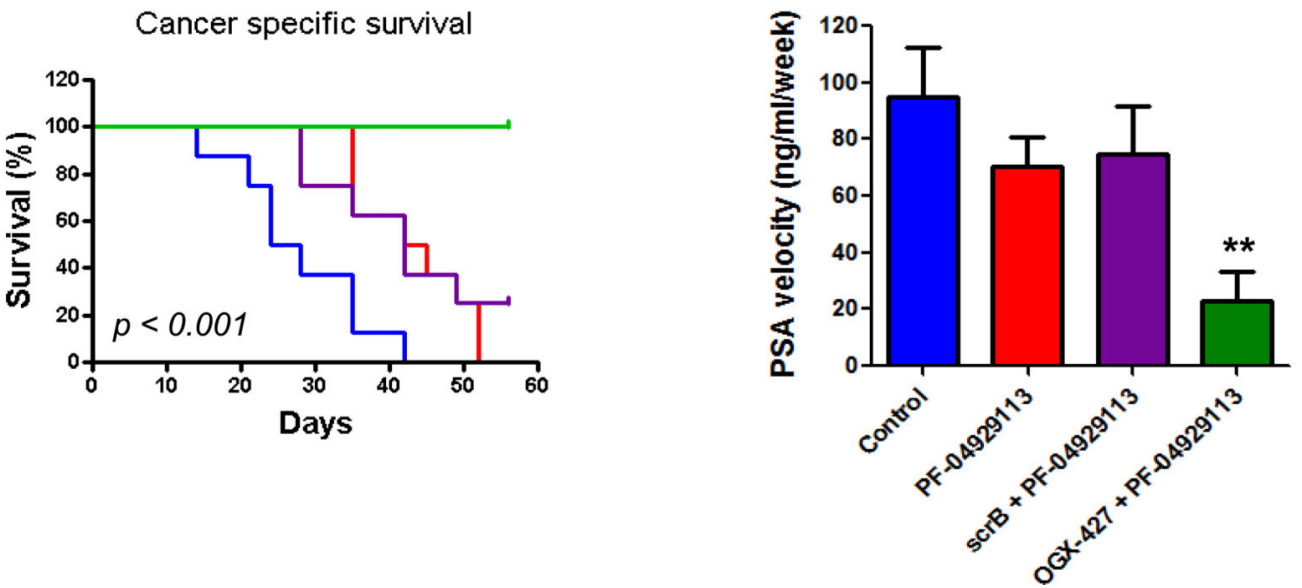

Fig. 5.

OGX-427 potentiates PF-04929113 activity in a LNCaP castrate-resistant prostate cancer xenograft model. (a) The mean tumor volume, (b) the percentage of tumor progression, and (c) the serum prostate-specific antigen (PSA) level were compared among the four groups plus or minus the standard error of the mean $(n=8)$. (d) PSA doubling time and velocity. (e) Using the Kaplan-Meier curve, cancer-specific survival was compared among the four groups over $56 \mathrm{~d}$. The control group corresponds to the mice bearing tumor that did not receive any treatment. This group only received vehicle. ${ }^{* * *} p<0.001$. 
A
B
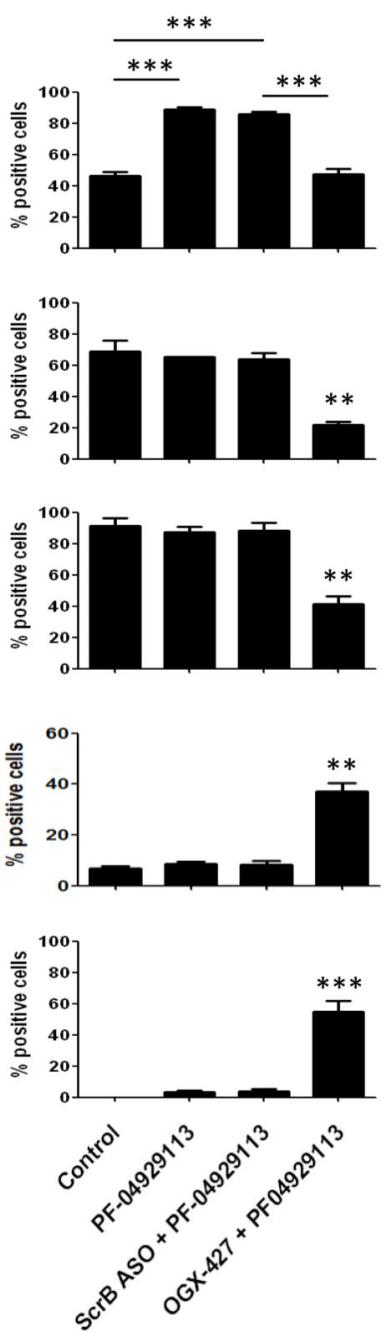

Fig. 6.

OGX-427 potentiates PF-04929113-induced apoptosis in castrate-resistant prostate cancer LNCaP tumors. (a) Tumors were collected after $56 \mathrm{~d}$ and heat shock protein (Hsp)27, Ki67, androgen receptor (AR), glucose-regulated protein (Grp) 78, and terminal deoxynucleotidyl transferase dUTP nick end labeling (TUNEL) were evaluated by immunohistochemical analysis (original magnification: $\times 200$ ). (b) Specimens were scored and estimated in percentage of positive cells. The control group corresponds to the mice bearing tumor that did not receive any treatment. This group only received vehicle. $* * * p<0.001$. 